\title{
Improved Elastic Registration of Low- Contrast Fluorescent Microscopy Images Using the Behaviour of Local Similarity
}

\author{
Mohsen Abbas Pour Seyyedi ${ }^{1}$ \\ m.abbaspourseyyedi@ecit.qub.ac.uk \\ Paul C. Miller ${ }^{1}$ \\ http://www.ecit.qub.ac.uk/ \\ Hugh Gribben ${ }^{2}$ \\ http://www.andor.com/
}

\author{
${ }^{1}$ ECIT at Queen's University Belfast \\ Belfast, UK \\ ${ }^{2}$ Andor ${ }^{\mathrm{TM}}$ Technology \\ 7 Millennium Way \\ Springvale Business Park \\ Belfast, UK
}

\begin{abstract}
We introduce a mechanism for improving the result of elastic registration for simultaneously acquired low-contrast fluorescent microscopy images. B-spline basis functions are commonly used for modelling nonrigid image deformations. We present a novel approach that improves the result of B-spline based registration process. This approach monitors the behaviour of local similarity measure values during optimization in order to recognize and exclude the pixels which drive the optimization into local optima. These are low intensity pixels, mostly in background regions but also in data areas, where there is no or little useful image structure to guide registration. Such pixels play a fallacious role in the registration process due to their high frequency content and pseudorandom contribution in the overall similarity measure and its derivatives. We show that excluding such pixels during the optimization considerably improves the registration result. This approach can reduce the average registration error up to several pixels and reduce the number of iterations down to one-third of the current implementations.
\end{abstract}

\section{Introduction}

Image registration $[1,2]$ is the process of finding a correspondence that maximizes a similarity measure between two or more deformed images. Elastic image registration, which handles warped deformations, is usually addressed as either a parametric modelling problem, based on B-splines [3,4], or solving the Euler-Lagrange partial differential equation using numerical methods [5]. The sum of squared differences (SSD) and mutual information (MI) are the most common similarity measures in use. The optimization methods are mostly a modified version of the Newton or the Gradient-Descent approaches. Recently, there has been more effort in evaluating registration results $[6,7]$.

Registration of microscope scans, especially simultaneously acquired low-contrast fluorescent microscopy images, has received little attention thus far. Recent confocal imaging techniques have been developed to utilise a per-pixel weighted subtraction on a pair of simultaneously acquired images. It is therefore necessary to ensure that the images are successfully registered before this processing step. Čapek et al. [8] expand the method of [3] in order to register 3-D eight-bit high-contrast confocal images. For low contrast

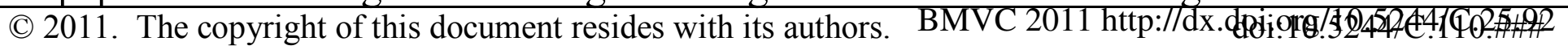
It may be distributed unchanged freely in print or electronic forms. 
microscopy images, the difference between data and background pixels is low. Therefore, the background pixels have a larger contribution to the overall similarity measure calculation. Such pixels can mislead the optimization process. Observations show that not only background pixels, but also some low-intensity data pixels can have a negative rather than positive effect on the optimization process. In addition, low-contrast images contain more high frequency components that cause greater sensitivity to interpolation artefacts [9]. In other words, because interpolation removes some details of the source image, it makes the similarity calculation inaccurate. So there is a higher chance of incorrect optimization. To the best of the authors' knowledge, such problems with low contrast images are not addressed in the image registration literature.

The optimization process is directed by the gradient of the similarity measure with respect to the B-spline coefficients. When there is not enough structure around a B-spline knot, the local similarity measures tend not to follow the same trend as the global value in approaching the optimal point, resulting in some coefficients being driven onto an incorrect path. This effect propagates through a number of surrounding knots which try to compensate for the fallacious ones. Therefore, there must be a dynamic mechanism for controlling the optimization process and excluding misleading pixels from the calculations. There are some landmarking approaches, such as pre-segmentation and contour detection, that only include certain pixels in the similarity calculation [10]. The success of such approaches depends on the accuracy of the landmarks. Regularization approaches, which try to preserve the objects in the image, are also used for improving image registration [3].

In this paper we present a novel mechanism that monitors the value of local similarity measure during registration and excludes pixels that mislead the optimization. We show that omitting such pixels during registration improves both accuracy and performance of image registration and produces faster convergence. In contrast to available methods, which treat the optimization as an input/output block, this approach constantly monitors what happens in each iteration step and prevents the deformation field from falling into incorrect local minima, which are sometimes irreversible. Section 2 describes B-spline based elastic registration and explains some of the problems with the low-contrast fluorescent microscopy images. Section 3 presents our novel algorithm. Experimental results are presented in section 4 , which are then followed by the conclusion in section 5 .

\section{Elastic registration of low contrast images}

In this section we describe B-spline based image registration. We also describe some drawbacks of using this approach with low-contrast microscopy images.

\subsection{B-spline based image registration for two images}

Consider two images $I_{t}(x, y)$ and $I_{s}(x, y)$, denoted target and source respectively, which are functions defined as mappings from the regions of interest $\Omega_{t} \subset \mathbb{Z}^{2}$ and $\Omega_{s} \subset \mathbb{Z}^{2}$ to the intensity domain $\mathbb{R}$. Image registration aims at finding a transformation function $\mathbf{T}(x, y): \mathbb{R}^{2} \rightarrow \mathbb{R}^{2}$ from the coordinates of $I_{t}$ to those of $I_{s}$. $\mathbf{T}$ can be modelled as a combination of an affine and an elastic transformation. In this paper we only consider the latter, which can be modelled using the piecewise cubic B-spline basis functions as in equation (1): 


$$
\mathbf{T}(x, y)=\sum_{k=0}^{3} \sum_{l=0}^{3}\left(\begin{array}{l}
c_{1, k, l} \\
c_{2, k, l}
\end{array}\right) \beta_{3}\left(\frac{x}{s_{x}}-k\right) \beta_{3}\left(\frac{y}{s_{y}}-l\right)
$$

In this equation, $\beta_{3}$ is a third-order B-spline basis function, $s_{x}$ and $s_{y}$ control the image resolution in the multiresolution registration framework, and $c_{1, k, l}$ and $c_{2, k, l}$ are the set of coefficients to be optimized. Many different implementations of the B-spline based image registration have been developed. In this paper we use the same implementation as the one presented in [11] and [12]. Preliminary experiments show that mutual information is more sensitive to interpolation artefacts when applied to sixteen-bit low contrast microscope images. This is because of the sparseness of occupied bins in the joint histogram. Therefore we use the average sum of squared differences (SSD) as the similarity measure which is defined in equation (2).

$$
S S D=\frac{1}{N_{\Omega}} \sum_{(x, y) \in \Omega}\left\{I_{t}(x, y)-I_{s}[\mathbf{T}(x, y)]\right\}^{2}
$$

Where $\Omega=\left\{(x, y) \in \Omega_{t}: \mathbf{T}(x, y) \in \Omega_{s}\right\}$ is a common mask between the two images and $N_{\Omega}$ is the number of pixels in the common mask.

\subsection{Problems of using SSD for low-intensity images}

SSD is used widely in image registration as it is easy to calculate, as are its derivatives. This makes it suitable for using with a broad range of interpolation and optimization algorithms. In low-contrast fluorescent microscopy images, the SNR level is low and the SSD value of background pixels is comparable to that of the data regions. Therefore, SSD of the background pixels will negatively affect the calculation of the overall SSD. In addition, using SSD as a similarity measure works best when the intensity range is the same for the source and target images. For high-contrast images, when the intensity ranges are different, adjusting the contrast of the images is a proper solution. In low-contrast microscopy images the intensity range is different, but because of the low SNR level, contrast adjustment enhances the background pixels too, which again has a negative effect on SSD calculation. For such images, the similarity measure, SSD, should only be calculated for those pixels in regions of well-defined data structure. In other words, we must exclude some of the pixels from the SSD calculation. Figure 2(c) is a good example of an image with valuable structure on small regions and background (pseudorandom noise) on all other regions. The B-spline based registration operates on local image regions, hence, in areas which lack structure, the algorithm will be misled. We can generalize this to the fact that, a perfect registration does not necessarily mean the minimization of the local SSD for all local regions (patches) in the image. Hub et al. [13] propose a method for evaluating image registration which assumes that the local values of SSD are also minimized for a good registration. We observed several low-contrast microscopy images which do not follow this assumption. For images like that of Figure 2(c), segmentation may seem an easy solution. However, registration is usually a preprocessing step and it is not desirable to have a segmentation step prior to registration. In addition, Figure 2(c) is an easy segmentation problem. As we will see later in this paper, the images may come in much more sophisticated topologies and intensities, making segmentation a separate challenging problem. 


\subsection{Patch space analysis}

Before we describe our methodology, we need to define the patch space since the local measurements are calculated over patches. For each coordinate $(x, y) \in \Omega$ in the image $I$, a patch $\mathcal{P}_{(x, y)}^{I}$ can be defined as the set of all pixels in a square of size $p \times p$ centred at $(x, y)$ where $p=2 q+1$ is an odd number. We can present the patch as a vector of size $p^{2}$ in the $p^{2}+2$ dimensional space of patches $\Gamma$, where the two additional dimensions represent the $x$ and $y$ coordinates [14]:

$$
\mathcal{P}_{(x, y)}^{I}=[I(x-q, y-q), \ldots, I(x+q, y+q)]
$$

\section{Registration based on fluctuations of the local SSD}

This section presents our novel algorithm for improving the B-spline based image registration. We analyse the behaviour of local SSD and describe how to use the insights gained to develop a faster and more robust image registration technique. In B-spline based image registration, the B-spline grid is deformed in the direction of the gradient of the SSD until the SSD is minimized. For a high contrast image, we expect that the SSD value of the local patches also converge to a minimum. As mentioned before, not all patches in low intensity microscopy images follow the same trend. In particular, the SSD of some patches shows a fluctuating behaviour. In other words, the SSD of a patch may increase and decrease in consecutive optimization iterations. There are different reasons for this behaviour:

1. Patches in the background regions show fluctuations in all iterations because calculating the SSD of such patches is equivalent to comparing two patches of random noise.

2. During the optimization, the data pixels in the source image find their perfect match coordinate in the target image. Not all data pixels are registered at the same time, but some converge to the optimal point quicker. When a pixel is registered, it starts showing fluctuations in the local SSD around the optimal point.

As mentioned before, for high contrast images, the SSD component due to the above fluctuations does not contribute significantly to the global SSD and its gradient. However, in low contrast microscopy images this component can drag the optimization into a wrong path. Therefore, we need a mechanism to exclude such misleading values. Our novel algorithm uses this behaviour to improve elastic registration as follows.

For each pixel $\left(x_{t}, y_{t}\right) \in \Omega_{t}$ in the target image $I_{t}$ with the corresponding patch $\mathcal{P}_{t,(x, y)}^{I}$, the local SSD is defined as in equation (4):

$$
S S D_{\mathcal{P}, t}=\frac{1}{p^{2}} \sum_{x=x_{t}-q}^{x_{t}+q} \sum_{y=y_{t}-q}^{y_{t}+q}\left\{I_{t}(x, y)-I_{s}[\mathbf{T}(x, y)]\right\}^{2}
$$

Because the optimization process is performed over time, we define the three dimensional space of SSD values $Z$. The members of $Z$ are the local SSD value for the patches $\mathcal{P}_{t,(x, y)}^{I}$ at iteration steps $\zeta$, where $\zeta=1,2,3, \ldots M$ represents the iteration number, with $M$ representing the final optimization iteration. The value of $M$ varies depending on 
the images and the algorithm in use. For each pixel $\left(x_{t}, y_{t}\right)$ and each iteration step $\zeta$ we define a stack of size $m$ of the most recent SSD values. This forms a two dimensional space $\Lambda_{\zeta}$ of vectors $\lambda_{\zeta, t,(x, y)}$ of length $m$, where $\lambda_{\zeta, t,(x, y)}$ is defined as in equation (5).

$$
\lambda_{\zeta, t,(x, y)}=\left[S S D_{\mathcal{P}, \zeta, t}, S S D_{\mathcal{P}, \zeta-1, t}, \ldots, S S D_{\mathcal{P}, \zeta-m+1, t}\right]
$$

The discrete time derivative of $\lambda_{\zeta, t,(x, y)}$ is equal to:

$$
D \lambda_{\zeta, t,(x, y)}=\left[d \lambda_{\zeta, t,(x, y)}, d \lambda_{\zeta-1, t,(x, y)}, \ldots, d \lambda_{\zeta-m+2, t,(x, y)}\right]
$$

Where:

$$
d \lambda_{\zeta-k, t,(x, y)}=S S D_{\mathcal{P}, \zeta-k, t}-S S D_{\mathcal{P}, \zeta-k-1, t}, \quad k \leq m-1
$$

If the number of consecutive sign changes in $D \lambda_{\zeta, t,(x, y)}$ is more than a certain value, which we call $\mu$, the algorithm excludes the pixel $\left(x_{t}, y_{t}\right)$ from further SSD calculation. For example, assume two pixels $\left(x_{1}, y_{1}\right)$ and $\left(x_{2}, y_{2}\right)$, with $m=6$ and:

$$
\left\{\begin{array}{l}
\operatorname{sign}\left(D \lambda_{\zeta,\left(x_{1}, y_{1}\right)}\right)=[-,+,-,+,-] \\
\operatorname{sign}\left(D \lambda_{\zeta,\left(x_{2}, y_{2}\right)}\right)=[+,+,-,+,-]
\end{array}\right.
$$

If $\mu=3$ for example, we exclude $\left(x_{2}, y_{2}\right)$ from further SSD calculation, but include $\left(x_{1}, y_{1}\right)$. When too many of the pixels are excluded from the optimization process, it is not suitable to continue the optimization because registration based on the remainder of the pixels is not reliable. We stop the optimization when fifty percent of the pixels are excluded. In section 4.3 we explain how we choose the important parameters.

\section{Results}

\subsection{Test data}

The proposed algorithm is applied to different microscopy images. The images are pairs of simultaneously acquired low contrast fluorescent microscopy slides which are called "left" and "right". The resolution of images is $1 \mu \mathrm{m}$ per pixel and the images are gray scale sixteen bit TIFF files of size $1040 \times 685$ pixels. The range of intensity for these images is between few hundreds and few thousands, which is small comparing to the maximum possible value in sixteen bit range. Figure 1 shows an example of low contrast microscope image pairs which is obtained from mouse kidney. Three pixels are highlighted in Figure 1(a). These pixels are analysed in section 4.5. Figure 2 shows six different sample images with different quality, contrast and topology. This figure only shows the "left" images. We call these images (a) to (f) for simplicity.

\subsection{Methodology}

In order to evaluate our method, we need a measure that can compare the registration result to a ground truth. We use the average registration error defined by Klein et al. [6] for 
comparing the registration result with a reference transform. The displacement error of a pixel $(x, y)$ is defined as follows. If the result of registration is a vector field $\boldsymbol{u}_{\text {reg }}(x, y)$ for each pixel in the source image, the displacement error is the difference between this vector and that of the reference transformation $\boldsymbol{u}_{r e f}(x, y)$, which is defined as: $D(x, y)=\left\|\boldsymbol{u}_{r e g}(x, y)-\boldsymbol{u}_{r e f}(x, y)\right\|$. When we evaluate the registration result for a patch $\mathcal{P}_{(x, y)}^{I}$ (or the entire image) with a region of interest $\Omega$, the average displacement error is defined as in equation (9):

$$
D_{\mathcal{P}_{(x, y)}^{I}}(\Omega)=\frac{1}{N_{\Omega}} \sum_{(x, y) \in \Omega}\left\|\boldsymbol{u}_{r e g}(x, y)-\boldsymbol{u}_{r e f}(x, y)\right\|
$$

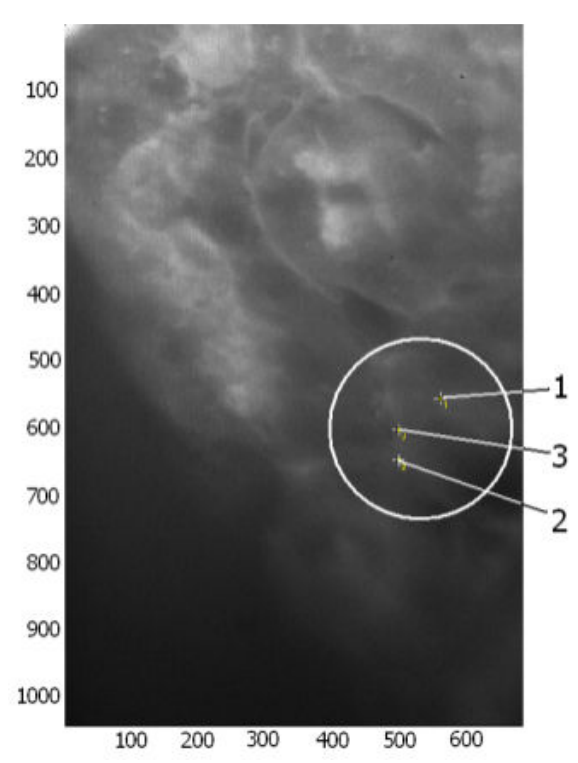

(a)

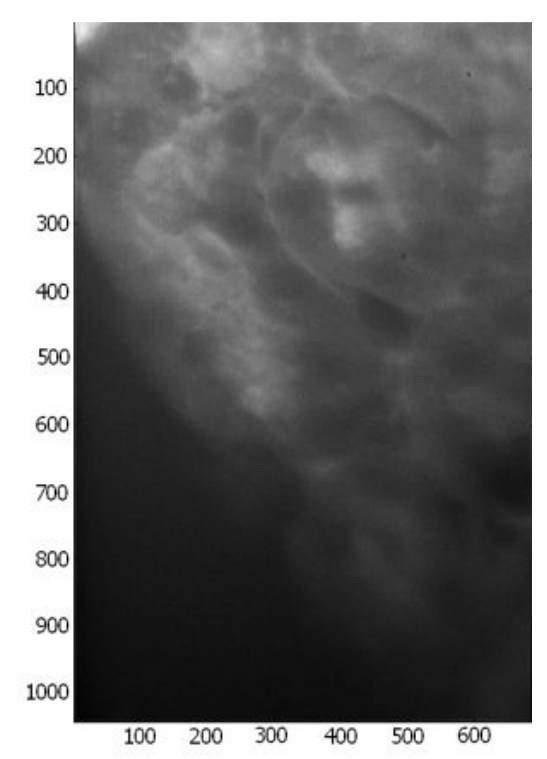

(b)

Figure 1: A sample image pair from mouse kidney: (a) Left image; (b) Right image.

This value is called the warping index in [3]. For obtaining the ground truth we apply a calibration approach based on using a high contrast reference pattern.

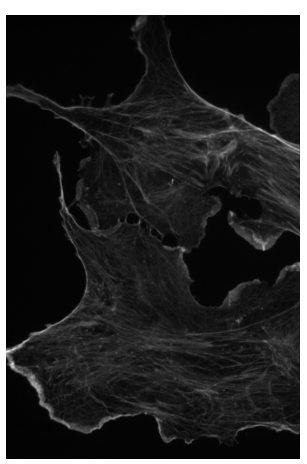

(a)

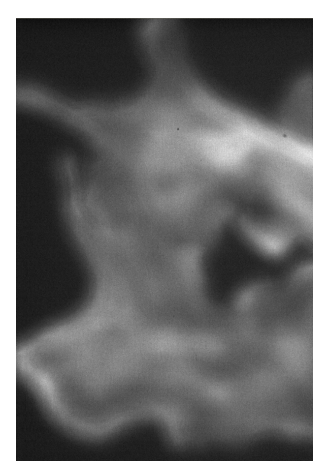

(b)

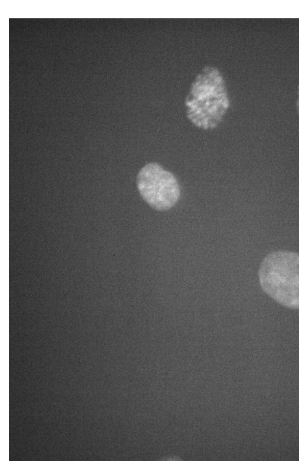

(c)

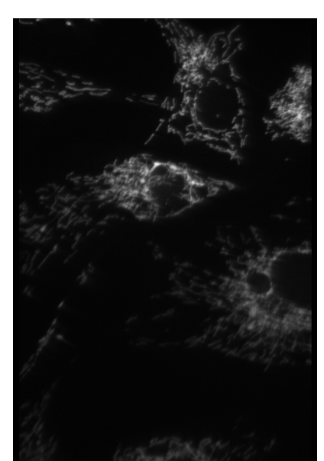

(d)

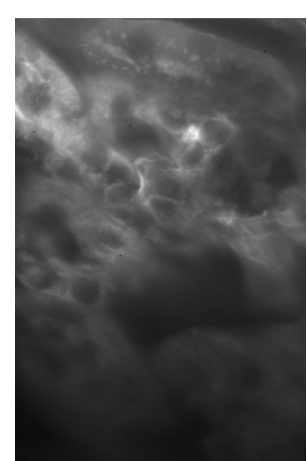

(e)

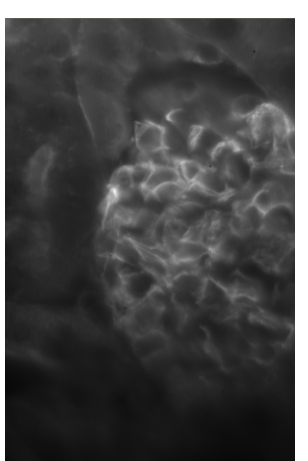

(f)

Figure 2: Image samples that are used in registration experiments.

\subsection{Investigation into patch size, stack size and $\boldsymbol{\mu}$ on performance}

In this section we study the effect of changing the main parameters, which are the patch size, stack size and $\mu$. We firstly predict an appropriate range for these parameters. Then we present the numerical experimental results to find the optimal values. The first important parameter is the patch size. For choosing a proper patch size, we notice that the 
fluctuations occur at coordinates where the gradient is not large and the optimization is not steep. Such smooth displacements in the B-spline knots can only cause deformations of a few pixels (one or two). Therefore, the patch size must be chosen in a way that can show enough variations with respect to such deformations. If the patch size is large, its SSD value would not show the expected fluctuations. Ideally, it is desirable to follow the SSD behaviour for individual pixels, i.e. $q=0$ in equation (4). However, the SSD of individual pixels can be sensitive to noise and interpolation artefacts. Values between $q=1$ and $q=4$ are expected to work better. B-spline based image registration is usually implemented in a multiresolution scheme, meaning that it starts with a small grid and a sub-sampled copy of the image and increases the grid and image resolution gradually [11]. This approach is called the pyramid approach, because the algorithm is applied to the grid and image in different resolutions in a pyramid form. In our algorithm we follow the multiresolution approach. Therefore, we need to increase the patch size with the increase in image pyramid level. The pyramid size in [11] increases with powers of two. However, if we use the same scaling for the patch size, the patch size for lower pyramid levels will be dramatically large. Instead, we prefer a linear increase in the patch size, meaning that, instead of using $p, p \times 2^{1}, p \times 2^{2}, \ldots$ we use $p$ in the top of the pyramid and $2 p, 3 p$, etc. for the next levels. The other important parameters are $m$ and $\mu$, which are the size of the SSD stack and the threshold of fluctuations in the local SSD for accepting a pixel. It is obvious that $m \geq 3$ and $\mu \leq m-2$. As mentioned before, the SSD in low contrast images is very sensitive to interpolation artefacts due to the presence of high frequency components. In other words, the fluctuation is a combination of the image content and the interpolation artefacts. Therefore, choosing a low value for $m$ can increase the risk of ignoring good pixels. Instead, choosing a larger stack size is safer. As a raw estimate we assume that half of the fluctuations are caused by artefacts and choose the stack size to be six.

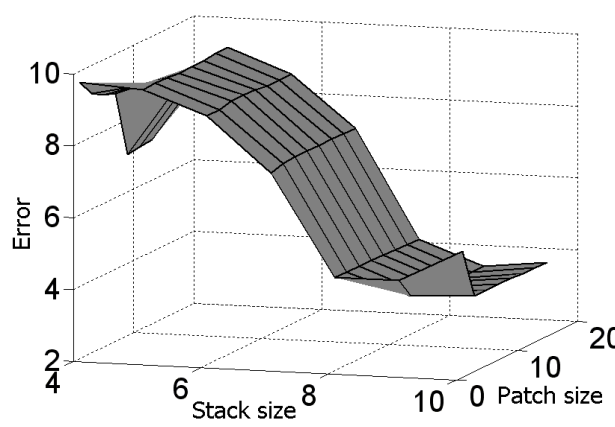

(a)

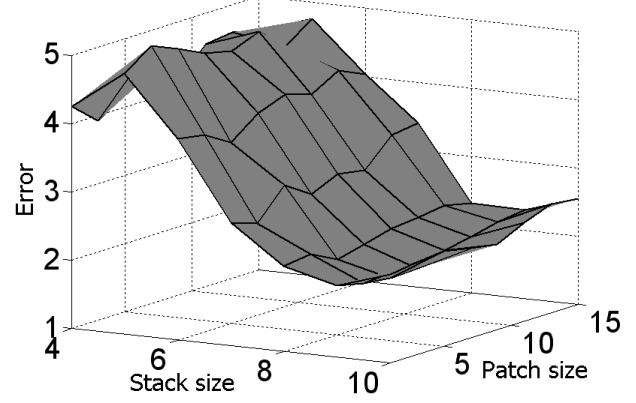

(d)

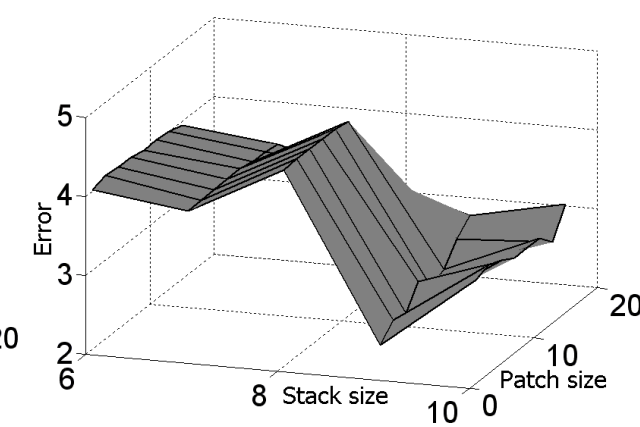

(b)

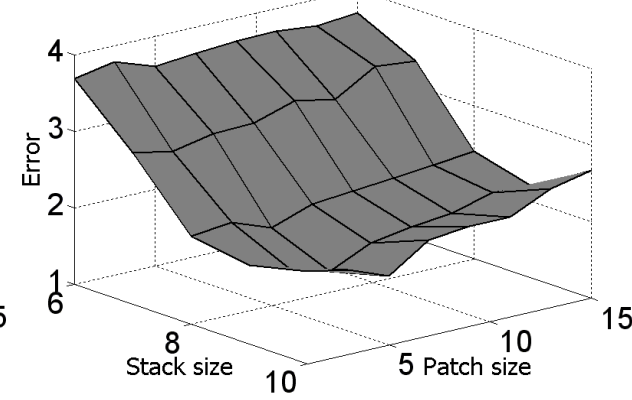

(e)

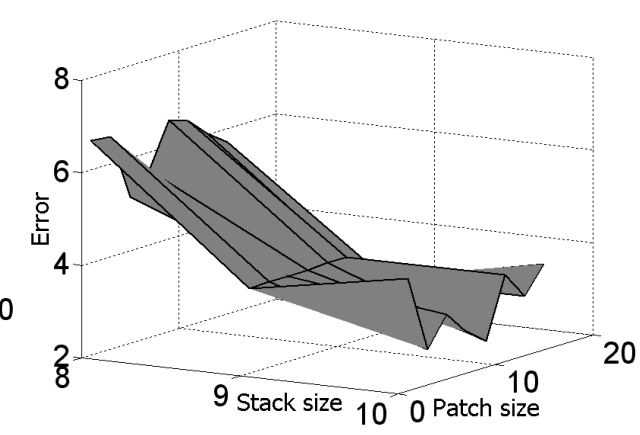

(c)

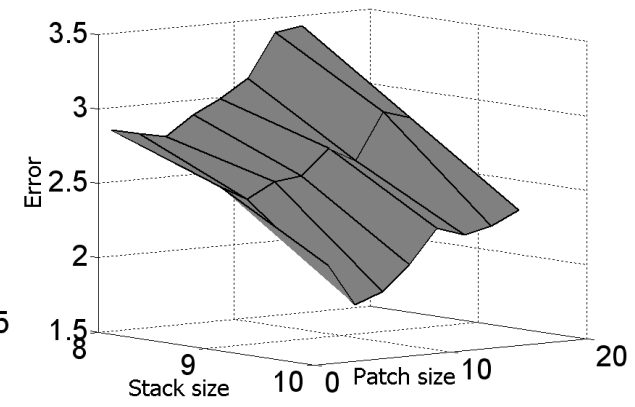

(f)

Figure 3: Average registration error as a function of $m$ and $p$ for different values of $\mu$ : (a) Figure 1 with $\mu=0$; (b) Figure 1 with $\mu=2$; (c) Figure 1 with $\mu=4$; (d) Figure 2(e) with $\mu=0$; (e) Figure 2(e) with $\mu=2$; (f) Figure 2(e) with $\mu=4$.

Figure 3 shows the value of average registration error for the two images in Figure 1 and Figure 2(e) for $\mu=0, \mu=2$ and $\mu=4$. When $\mu$ is small (zero or one), the value of error is 
large for small stack size, which means a poor registration. The error is worse than the current approach which uses all pixels. This conforms to our prediction about the value of $\mu$. A small $\mu$ means that we are excluding many of the useful pixels, which are probably fluctuating only because of the interpolation artefacts. For $\mu=2$ the error seem to be more independent of $\mu$. Figures 3(c) and 3(f) $(\mu=4)$ suggest that for a larger stack size, the error depends more on the difference between stack size and $\mu(m-\mu)$ than the value of $\mu$ alone. Several experiments confirm that $m=7$ and $\mu=3$ is a good combination. As we see in Figure 3, the error value is not much dependent on the patch size. However, increasing the patch size slightly reduces the error. Other experiments show that the error will increase considerably for $p \geq 25$. In most experiments, the registration error converges to a minimum value.

Figure 4 shows the number of iterations for the same experiment for the image in Figure 1. We see that for a small $\mu$, the number of iterations is very small, which corresponds to poor error values. This means that the algorithm is stopped sooner than it is optimized, because many good quality pixels are excluded from SSD calculation. With larger values of $\mu$, the number of iterations again depends more on the difference between stack size and $\mu$. With a larger difference, the error is more trustable and the number of iterations is larger. The pattern of change in the error and number of iterations is similar for most of the images.

\subsection{Results of registration}

Now we analyse the behaviour of a few individual pixels to investigate how the fluctuations in the local SSD of individual pixels can affect the overall SSD calculation and optimization. We compare the results of our approach to those of a state of the art Bspline based implementation and show how excluding the poor quality pixels can improve both quality and performance of registration.

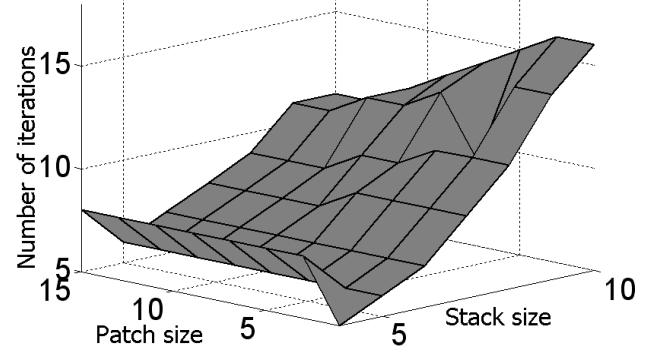

(a)

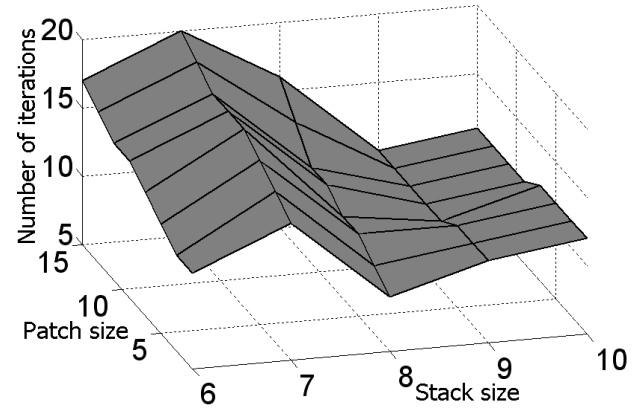

(b)

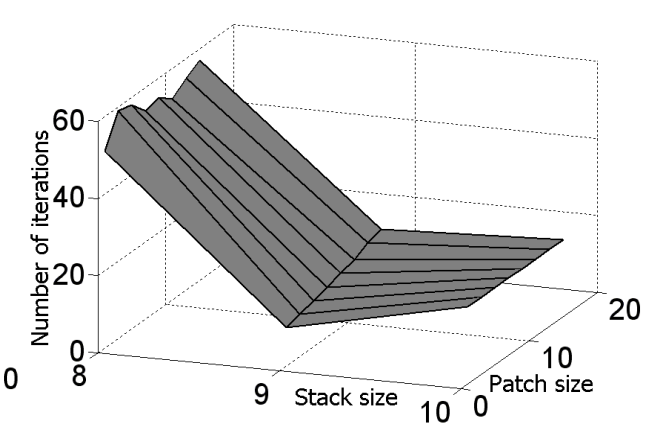

(c)

Figure 4: Number of iterations as a function of $m$ and $p$ for different values of $\mu$ : (a) Figure 1 with $\mu=0$; (b) Figure 1 with $\mu=2$; (c) Figure 1 with $\mu=4$.

Figure 5(a) shows the normalized value of the SSD for the three pixels that are landmarked in Figure 1(a). Pixel number one is recognized as a good pixel using our algorithm, because its fluctuations are few and it lasts the excluding process until the final optimization iteration. Pixels two and three on the other hand, fail to stay in the optimization because of their fluctuating local SSD. As we see in Figure 5(a), the local SSD of the "good" pixel follows the minimization trend, while the two others do not converge. The trend of the local SSD for the good pixel is similar to that of the overall SSD in Figure 5(b). This figure shows the overall SSD value for both the image registration algorithm of [12] and our approach. In Figure 6 we show how the pixels are 
excluded from the registration process. As mentioned before, after the first few iteration steps, the pixels that do not have enough image structure are excluded. These pixels usually belong to background regions, but they can belong to data regions as well. As the optimization goes on, more and more pixels are being masked from registration. Figure 6(a) shows the first mask which is extracted after 7 iterations mask. Figure 6(b) shows an intermediate mask, after 28 steps.

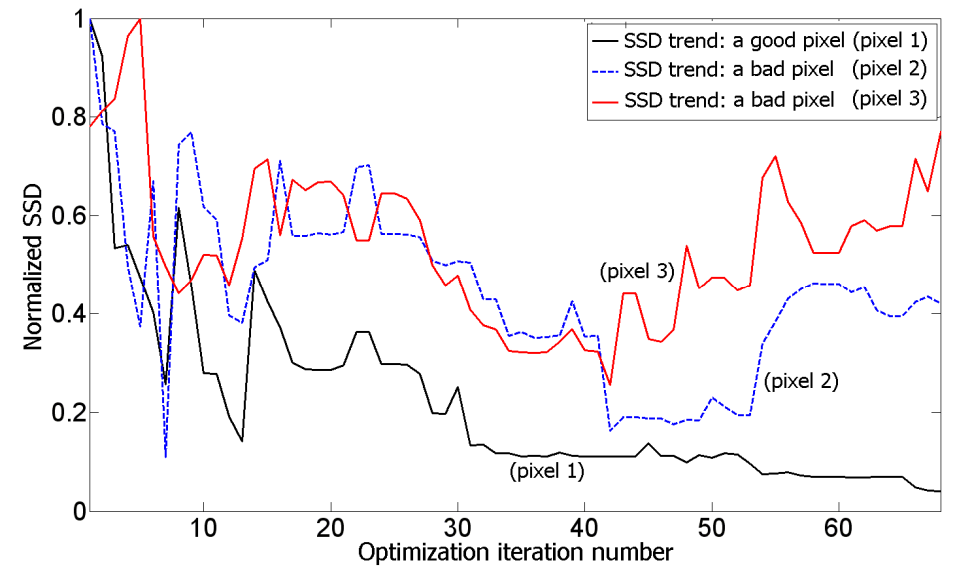

(a)

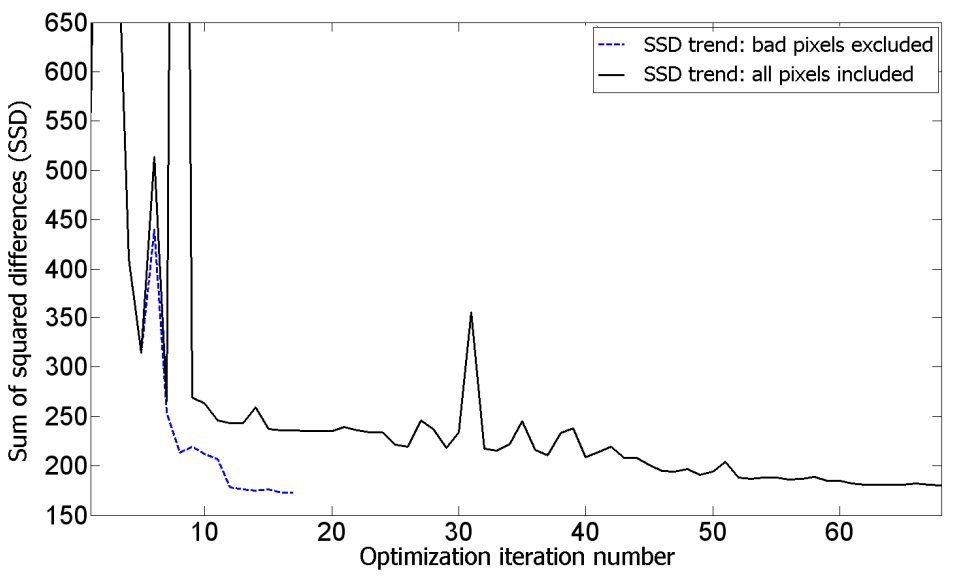

(b)

Figure 5: Local and overall SSD trend: (a) Normalized local SSD for the three pixels in Figure 1(a) before applying our approach; (b) overall SSD for the mouse kidney image of Figure 1 before and after excluding poor quality pixels.

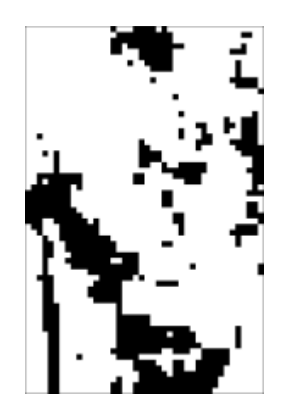

(a)

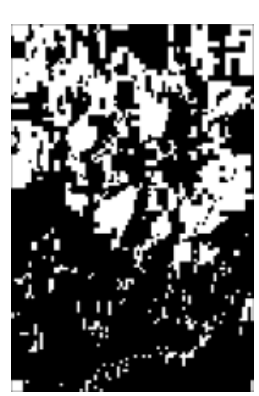

(b)

Figure 6: Registration mask after (a) 7 iterations; (b) 28 iterations.

Our algorithm is developed as an improvement to the implementation of the B-spline based image registration [12]. Therefore, we compare it to our approach. We also compare our results to those of another approach [3, 15], which introduces a consistent and regularized registration. Table 1 shows the average registration error for the images in Figure 1 and Figure 2. Table 2 shows the number of iteration steps in the same experiment. In these experiments, the Patch size $p$ is five, the stack size $m$ is seven and $\mu=3$. As we see in Table 1, in all experimental results the registration error improves. For images with less structure, the improvement is up to several pixels. At the same time, the number of iterations decreases considerably (Table 2) which means a faster convergence of the registration algorithm. As mentioned before, the reason for this result is that, many of the pixels, mainly belonging to the background but also pixels of the data regions do not provide a valuable contribution to the registration process. Instead, they cause unnecessary fluctuations in the value of the overall SSD and make the registration deviate from the correct optimization path. Examples of such pixels are presented in Figure 5(a). We solve this problem via a dynamic recognition and exclusion of such pixels. This helps the optimization algorithm converge faster. At the start of the algorithm, the most unreliable pixels are excluded. During optimization, more and more pixels converge to the optimal point so they must be excluded from further registration. One of the main approaches for 
improving image registration is preserving the topology of the image via some regularization [3,4] methods. As we see in Table 1 and Table 2, this method cannot improve the results for low-contrast confocal images because it only handles the regions with image structure and does not provide any solution for regions without enough structure. The same way, the consistent image registration [15] suffers from the same problem.

\begin{tabular}{|l|ccccccc|}
\hline Image sample & Figure1 & (a) & (b) & (c) & (d) & (e) & (f) \\
\hline \hline Poor pixels excluded & 3.64 & 1.41 & 4.99 & 1.09 & 10.68 & 1.70 & 1.16 \\
All pixels included & 6.20 & 0.87 & 5.44 & 1.33 & 15.72 & 2.06 & 1.84 \\
Consistent and regularized & 6.02 & 6.49 & 5.24 & 0.83 & 29.07 & 2.15 & 1.59 \\
\hline
\end{tabular}

Table 1: Average registration error for images of Figure 1 and Figure 2 for three approaches: excluding poor quality pixels, using all pixels [12] and the consistent and regularized approach $[3,15]$.

\begin{tabular}{|l|ccccccc|}
\hline Image sample & Figure1 & (a) & (b) & (c) & (d) & (e) & (f) \\
\hline \hline Poor pixels excluded & 20 & 27 & 59 & 29 & 51 & 24 & 33 \\
All pixels included & 68 & 41 & 54 & 36 & 106 & 65 & 54 \\
\hline
\end{tabular}

Table 2: Number of iterations for registering the images of Figure 1 and Figure 2 for two approaches: excluding poor quality pixels, using all pixels [12].

\section{Conclusion}

We presented a new image registration approach that calculates the similarity measure and its gradients using only valuable pixels. Image registration algorithms usually calculate the similarity measure over the entire image, regardless of the importance of different pixels. In simultaneously acquired low-contrast fluorescent microscopy images, the value of similarity measure for background regions and poor quality pixels is comparable to that of the data regions. This effect can drag the optimization algorithm out of the correct path. Regularization based approaches suffer from the same problem when applied to lowcontrast images. Our method is not based on segmentation or landmarking in the image, but extracts and ignores the misleading pixels during the optimization. In contrast with the common methods, this approach does not treat the optimization task as an input-output block, but monitors it constantly. We presented the results of applying this algorithm to several low-contrast microscopy images and showed that the registration error is reduced up to several pixels. In addition, our method reduces the number of iterations down to onethird of the original implementation in [12]. The future work may include more investigation of the effect of the parameters, taking a closer look at different values of local similarity measure and improving the mechanism by which the poor quality pixels are being excluded. We also need to present the error for the "good" pixels explicitly, which shows the strength of our approach much better. 


\section{References}

[1] B. Zitova and J. Flusser. Image Registration Methods: A Survey. Image and Vision Computing, 21: 977-1000, 2003.

[2] A. Gholipour, N. Kehtarnavaz, R. Briggs, M. Devous, and K. Gopinath. Brain Functional Localization: A Survey of Image Registration Techniques. IEEE Transactions on Medical Imaging, 26(4): 427-451, 2007.

[3] C. Ó. S. Sorzano, P. Thévenaz, and M. Unser. Elastic Registration of Biological Images Using Vector-Spline Regularization. IEEE Transactions on Biomedical Engineering, 25(4): 652-663, 2005.

[4] D. Rueckert, L. I. Sonoda, C. Hayes, D. L. G. Hill, M. O. Leach, and D. J. Hawkes. Nonrigid Registration Using Free-Form Deformations: Application to Breast MR Images. IEEE Transactions on Medical Imaging, 18(8): 712-721, 1999.

[5] G. Hermosillo, C. Chefd'Hotel, and O. Faugeras. Variational Methods for Multimodal Image Matching. International Journal of Computer Vision (Springer), 50(3): 329-343, 2002.

[6] S. Klein, M. Staring, and J. P. W. Pluim. Evaluation of Optimization Methods for Nonrigid Medical Image Registration Using Mutual Information and B-Splines. IEEE Transactions on Image Processing, 16(12): 2879-2890, 2007.

[7] J. Kybic, "Bootstrap Resampling for Image Registration Uncertainty Estimation Without Ground Truth," IEEE Transactions on Image Processing, 19(1), 64-73, 2010.

[8] M. Čapek, P. Brůža, J. Janáček, P. Karen, L. Kubínová, and R. Vagnerová. Volume Reconstruction of Large Tissue Specimens from Serial Physical Sections Using Confocal Microscopy and Correction of Cutting Deformations by Elastic Registration. Microscopy Research and Technique, 72: 110-119, 2009.

[9] G. K. Rohde, D. M. Healy, Jr., C. A. Berenstein, and A. Aldrouhi. Interpolation Artifacts in Biomedical Image Registration. In Proc. IEEE Int. Symposium on Biomedical Imaging: From Nano to Macro, 648-651, 2009.

[10] M. V. Wyawahare, P. M. Patil, and H. K. Abhyankar. Image Registration Techniques: An overview. Int. Journal of Signal Processing, Image Processing and Pattern Recognition, 2(3): 11-28, 2009.

[11] J. Kybic, P. Thévenaz, A. Nirkko, and M. Unser. Unwarping of Unidirectionally Distorted EPI Images. IEEE Transactions on Medical Imaging, 19(2): 80-93, 2000.

[12] J. Kybic, and M. Unser. Fast parametric elastic image registration. IEEE Transactions on Image Processing, 12(11), 1427-1442, 2003.

[13] M. Hub, M. L. Kessler, and C. P. Karger. A Stochastic Approach to Estimate the Uncertainty Involved in B-Spline Image Registration. IEEE Transactions on Medical Imaging, 28(11): 1708-1716, 2009.

[14] D. Tschumperlé and L. Brun. Image Denoising And Registration by PDE's on The Space of Patches. Int. Workshop on Local and Non-Local Approximation in Image Processing (LNLA'08), 2008.

[15] I. A. Carreras, C. Sorzano, R. Marabini, J. Carazo, C. O. De Solorzano, and J. Kybic. Consistent and Elastic Registration of Histological Sections Using Vector-Spline Regularization. Computer Vision Approaches to Medical Image Analysis, 85-95, 2006. 